\title{
Evaluation de la circoncision réalisée en ambulatoire chez l'adulte : impact sur les M.S.T. et sur la sexualité
}

\author{
M.H. LIBERT *, T. HURARD *, M. DEPIERREUX ** \\ *Urologie ; **Anatomo-pathologie - Centre Hospitalier César de Paepe, Bruxelles
}

Cet article a été l'objet d'une présentation sous forme de panneau affiché à la 2ème journée scientifique de la SALF, Paris, 18 Juin 1994.

\section{RESUME}

Nous avons évalué par un questionnaire l'impact sur les MST et la sexualité de la circoncision pratiquée chez des patients adultes pour diverses indications. L'indication la plus fréquente (75\%) était représentée par des MST rebelles ou récidivantes. Dans les suites, près de $96 \%$ des patients se sont déclarés satisfaits de leur sexualité ; près de $80 \%$ ne signalent pas de récidive de leur affection.

Mots-clés : Circoncision, maladies sexuellement transmissibles, rapports sexuels.

Que représente pour un homme adulte, sexuellement actif, la réalisation d'une circoncision? S'agit-il d'une opération subie et vécue comme toute autre ou, au contraire, la composante émotionnelle et sexuelle y joue-t-elle un rôle plus important? La modification esthétique et tactile a-t-elle une conséquence sur la sexualité, amène-t-elle une modification dans la fréquence et l'évolution des M.S.T. ?

Nous avons tenté d'apporter des éléments de réponse à ces questions par le biais d'un questionnaire (Tableau 1) adressé de façon anonyme à une cinquantaine de patients adultes qui ont subi une circoncision sous anesthésie locale, en ambulatoire. A ce questionnaire, adressé au moins 2 mois après la circoncision, 24 patients ont répondu, la plupart satisfaits de l'opération. Ces $48 \%$ de réponses sont-ils indicatifs de la réalité ou, au contraire, ce score indique-t-il une importante proportion d'indifférents ou de mécontents? Notre analyse, qui ne peut se baser évidemment que sur l'évaluation des réponses reçues, rejoint certains des témoignages rapportés par M. CHÉBEL [1].

L'âge moyen était de 40,7 ans (de 18 à 78 ans). Dans $75 \%$ des cas, le motif de la circoncision était en relation avec des M.S.T. rebelles ou récidivantes (condylomes, dysplasies, mycoses, balanites) ou des lésions scléreuses invalidant les relations sexuelles (phimosis partiel ou complet, lichen scléroatrophique). Pour quelques patients, l'indication correspondait à des difficultés d'hygiène ou à un souhait personnel (Tableau 2). La répartition des lésions histologiques est évoquée au Tableau 3.

\section{CRITERES D'INCLUSION}

- Age $>18$ ans.

- Activité sexuelle régulière avant lintervention.

- Circoncision réalisée sous anesthésie locale et en ambulatoire.

- Pas de motif religieux à l'origine de la circoncision.

\section{TECHNIQUE}

- Anesthésie par injection unique de $10 \mathrm{ml}$ de xylocaïne $2 \%$, en bague, à la base de la verge. 
Age au moment de la circoncision :

Recul actuel depuis l'opération :

mois/années

Etes-vous d'origine

$\begin{array}{ll}\text { européenne } & 0 \\ \text { américaine } & 0 \\ \text { arabe } & 0 \\ \text { autre } & 0\end{array}$

La circoncision a été réalisée pour :

$\begin{array}{lc}\text { infection récidivante } & 0 \\ \text { prépuce trop étroit } & 0 \\ \text { éjaculation précoce } & 0 \\ \text { hygiène } & 0 \\ \text { à ma demande, pour raison personnelle } & 0 \\ \quad \text { pour motif religieux } & 0\end{array}$

\section{Actuellement :}

L'éjaculation est

$\begin{array}{llll}\text { plus retardée } & 0 & \text { plus satisfaite } & 0 \\ \text { inchangée } & 0 & \text { moins satisfaite } & 0 \\ \text { plus précoce } & 0 & \text { sans opinion } & 0\end{array}$

Si vous aviez des infections avant la circoncision, celles-ci sont maintenant :

$\begin{array}{ll}\text { absentes } & 0 \\ \text { moins fréquentes } & 0 \\ \text { pas de changement } & 0\end{array}$

Depuis la circoncision, la qualité globale des rapports sexuels est :

$\begin{array}{ll}\text { améliorée } & 0 \\ \text { inchangée } & 0 \\ \text { altérée } & 0\end{array}$

En résumé:

Je suis satisfait

o

je regrette d'avoir réalisé cette opération

0

Tableau 2 : Indication de la circoncision. NB : Certains patients répondent à plusieurs indications.

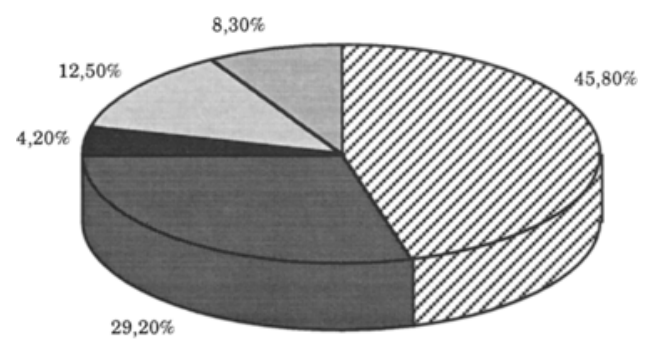

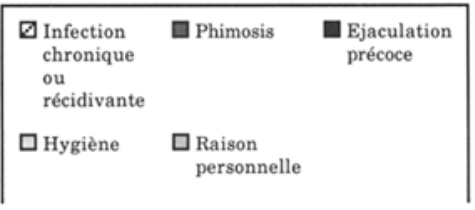

Tableau 3 : Lésions histologiques.

- absence de lésions microscopiques : 1

- inflammation chronique aspécifique : 11

- infection à papillomavirus (condylomes et/ou macules planes), sans signes histologiques de dégénérescence : 7

- infections à papillomavirus avec signes de dégénérescence (PIN I ou II) : 1

- épithélioma in situ : 1

- lichen scléro-atrophique : 3 
Tableau 4A : Taux de satisfaction globale du patient.
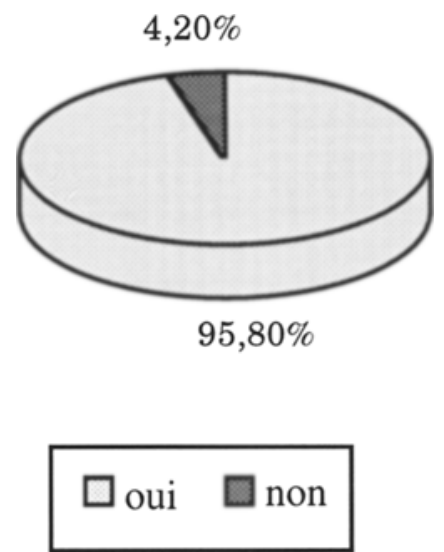

Tableau 5 : Evolution de la qualité des rapports sexuels après circoncision.

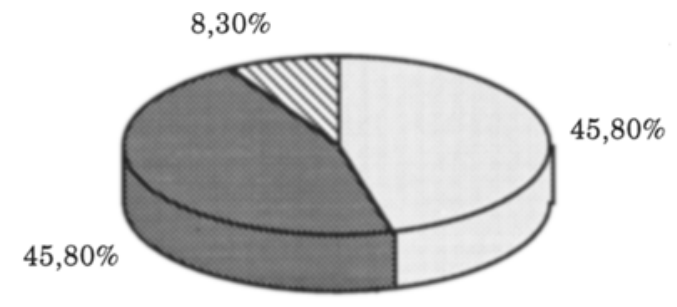

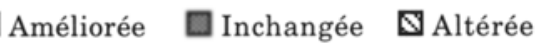

Tableau 7 : Conséquences sur l'éjaculation.

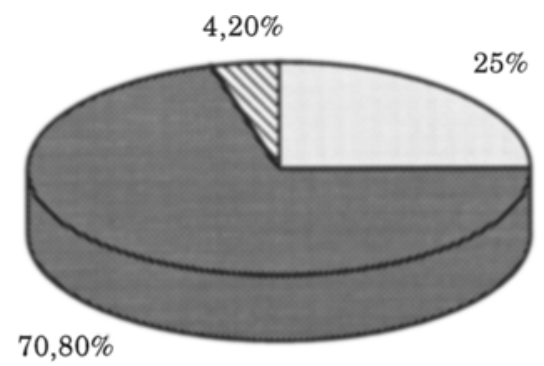

Retardée $\square$ Inchangée $\quad$ Plus précoce
Tableau 4B : Infections après la circoncision.

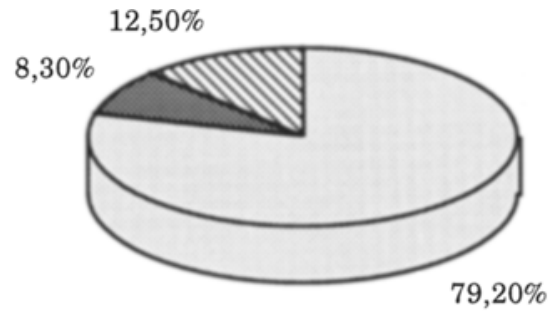

Absentes

Moins
fréquentes

Réponse sans objet

Tableau 6 : Avis de la (du) partenaire.

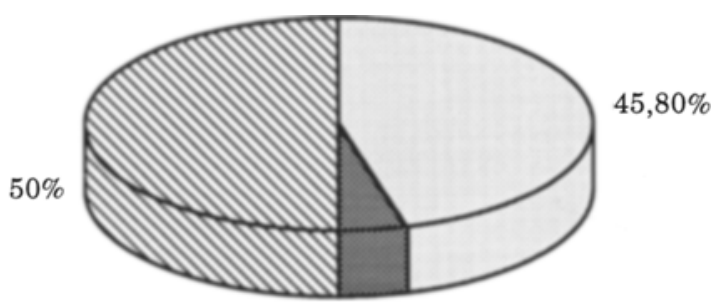

$4,20 \%$
Rapport sexuel plus satisfaisant
Rapport sexuel moins satisfaisant
Q Rapport sexuel inchangé
- Circoncision classique, hémostases au bistouri électrique, suture par points séparés de catgut $3 / 0$ ou Vicryl ${ }^{\circledR}$ rapide $3 / 0$.

- Tulle gras et pansement légèrement compressif pour $24 \mathrm{H}$.

- Soins à domicile : Lavage quotidien (eau claire). Séchage (sèche-cheveux). Mercurochrome (solution aqueuse).

\section{RESULTATS}

Globalement, 23/24 (95,8\%) patients sont satisfaits de leur sexualité et des conditions d'hygiène après l'intervention. La majorité d'entre eux ne présentent plus 
d'infections $(79,2 \%)$ ou signalent des infections moins fréquentes $(8,3 \%)$; cette question est sans objet pour les autres patients (pas d'infection avant ni après la circoncision). A ce propos, l'évaluation est donc nettement positive (Tableaux $4 \mathrm{~A}$ et 4B).

Pour $45,8 \%$ des patients, la qualité des rapports sexuels est améliorée par la réalisation de la circoncision, la même proportion de patients estime qu'il n'y a aucune modification remarquable ; $8,3 \%$ ne sont pas satisfaits (Tableau 5).

Quant aux partenaires, 96\% (soit 23/24) sont plus satisfaites $(46 \%)$ ou indifférentes $(50 \%)$. Le seul avis négatif émane d'un partenaire au sein d'une relation homosexuelle (Tableau 6).

Enfin, pour 17 patients (71\%), la circoncision et la modification de l'aspect du frein qui en découle généralement n'ont pas d'influence sur la qualité ou la rapidité de l'éjaculation. Dans le groupe des éjaculateurs précoces (6 patients, $25 \%$ des cas), l'amélioration se caractérise par une latence plus grande. Un seul signale une éjaculation plus précoce (Tableau 7).

\section{CONCLUSIONS}

Les réponses mettent en évidence :

1. La bonne tolérance de cette chirurgie, sous anesthésie locale et ambulatoire.
2. La résolution définitive, dans tous les cas, du problème de MST à l'origine de la décision de circoncision.

3. La qualité maintenue ou améliorée des rapports sexuels, plus de $90 \%$ des patients étant satisfaits et ne regrettant pas leur décision.

\section{REFERENCE}

1. Chebel $M$ : Histaire de la circoncision, des origines à nos jours. p 29-36, Le Nadir, Balland, Ed. Paris, 1992.

\section{ABSTRACT}

\section{Assessment of circumcision practiced on adult}

M.H. Libert, T. HuRARD, M. DepierReuX

With the help of a questionnaire, we assessed the impact of circumcision, practiced on adult patients for various indications, on sexually transmitted diseases (STD) and sexuality. Recurrent or obstinate STD represented the most frequent indication (75\%). Subsequentely almost $96 \%$ of the patients declared to be satisfied with their sexuality and nearly $80 \%$ did not mention any recurrence of their disease.

Key words : Circumcision, sexually transmitted diseases, sexual intercourse. 